\section{Influenza Vaccination: Impact of an Intervention Campaign Targeting Hospital Staff}

TO THE EDIT OR-Between 1984 and 2002, the morbidity impact of influenza in France averaged 2.9 million cases per season-that is, $1 \%-8 \%$ of the population was affected. ${ }^{1} \mathrm{Im}$ munization against influenza is officially recommended for all persons older than 65 years of age and for patients with chronic disease regardless of age; the cost of the vaccine is paid by the French national social security system. Vaccination is also recommended for persons liable to spread the virus: caregivers, school teachers, and anyone in regular or prolonged contact with at-risk persons. ${ }^{2}$ However, among caregivers, vaccination coverage ranges from $4 \%$ to $10 \%$ of staff. ${ }^{3,4}$ In Nice University Hospital, where vaccination is provided free of charge by the occupational medicine department, only $5.3 \%$ of 6,600 employees received the vaccine in 2001. We tested the feasibility of an active intervention to raise awareness among caregivers about nosocomial influenza prophylaxis, which was conducted by a mobile medical team offering information and on-the-spot immunization, and evaluated its impact on vaccination coverage.

The intervention took place between October and December 2002 among the staff of 6 departments in Nice University Hospital that care for patients at risk of complications related to influenza or patients among whom the prevalence rate of influenza is expected to be high during an epidemic (ie, patients in medical intensive care unit, the hematology unit, the internal medicine wards, the pediatric ward, and outpatient clinics, and patients undergoing abdominal surgery or hepatic transplan- tation). Information sessions were directed at all staff in regular contact with patients: head nurses, senior and student nurses, practical nurses, orderlies, and cleaning staff. Sessions were scheduled in order to recruit both daytime and nighttime staff on a voluntary basis. Sessions consisted of a slide show that explained the issue of nosocomial influenza and provided objective arguments in response to any doubts, queries, and preconceptions concerning influenza immunization; this was followed by an informal discussion, and vaccination was offered on the spot, after controlling for contraindications.

At the end of the epidemic period, a questionnaire was distributed to all the participants, regardless of their vaccination status, to gather the following information: history of influenza-like illness in the past and during the 2002-2003 season, history of vaccination during the previous and present years, reasons for acceptance or refusal of immunization, any adverse events, intention to receive vaccination the following year, statements about benefits of influenza vaccination, statements about the impact of current debates concerning certain vaccines on the decision to receive influenza immunization, and knowledge acquired during the information sessions (based on a brief assessment). Univariate analyses of findings were performed with the Fisher exact test, Mann-Whitney $U$ test, Student $t$ test, and Kruskall-Wallis test.

Fifty 25-45-minute sessions took place during the days October 16 through December 5, 2002. Of 405 targeted staff, $58 \%$ attended one of the presentations. Response was higher during night shifts, when staff were likely more available. Another 35 nontargeted persons also attended and were therefore included in the analysis. Among those who attended, 57\% decided to receive influenza vaccine; that is, $61 \%$ of the total cohort, taking into account those who had already been vaccinated through their own initiative prior to the intervention,

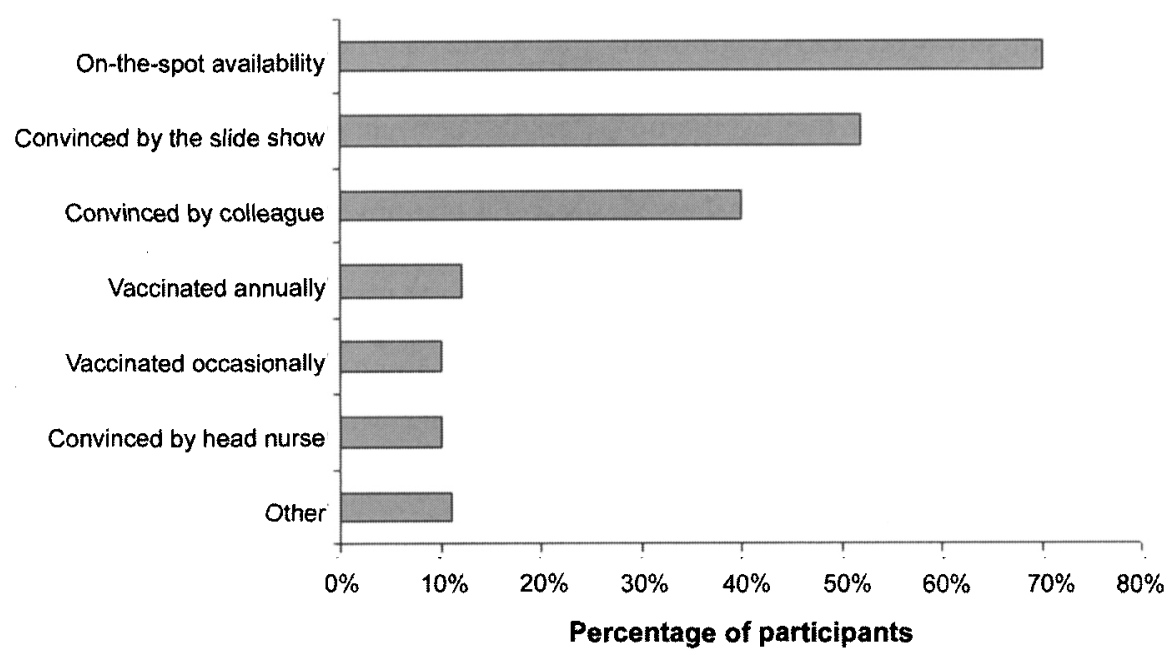

FIGURE. Reasons participants gave for accepting immunization 
TABLE. Comparison of Clinical Characteristics of Vaccinated and Nonvaccinated Participants

\begin{tabular}{lccc}
\hline & \multicolumn{2}{c}{ Percentage of participants } \\
\cline { 2 - 3 } Characteristic & $\begin{array}{c}\text { Vaccinated } \\
(n=118)\end{array}$ & $\begin{array}{c}\text { Not vaccinated } \\
(n=63)\end{array}$ & $P$ \\
\hline Past history of influenza & 54 & 32 & .006 \\
Prior immunization against influenza & 33 & 8 & .0002 \\
Influenza or flulike syndrome in 2002-2003 & 13 & 19 & NS \\
Work days lost due to influenza or flulike syndrome & 2 & 10 & .014 \\
Suspicious of vaccination because of debates about & & & .0028 \\
\hline
\end{tabular}

NOTE. NS = not significant.

and $35 \%$ of the theoretical overall number of targeted staff numbers. Vaccination rates were higher among nurse supervisors and senior nurses, night duty personnel and intensive care staff. Prior to our awareness-raising sessions, the annual rate of influenza vaccination among the staff included in our survey was $7 \%$. Sartor et al..$^{5}$ reported a similar increase in vaccination rates after implementation of a mobile-cart program in a French hospital.

In April 2003, sixty-seven percent of questionnaires sent to the staff members and students who attended the sessions were completed and returned. Sixty-five percent of respondents had been vaccinated during the intervention. Reasons for accepting vaccination are shown in the Figure, and comparison of vaccinated and nonvaccinated respondents regarding past and present experience of influenza and vaccination are summarized in the Table. Most respondents said they intended to seek vaccination the next year, preferably if they could be vaccinated within the hospital department.

The positive impact of our intervention resulted from 2 essential features: provision of information, and easy access to vaccination. Many participants ignored the scientific information on viral diseases and their dangers. Those who were vaccinated knew more about influenza than the nonvaccinated group, suggesting that properly integrated information improves the likelihood of acceptance of vaccination. Lack of time or motivation, which was explicitly mentioned by 9 respondents, may explain why the vaccination rate was low even though vaccination was provided free by the occupational medicine department. Difficulties in involving hospital staff have been described by Yassi et al., who showed that only $36 \%$ of those who were favorable to influenza vaccination were actually vaccinated.

The beneficial role of the head nurse in promoting vaccination is shown by the fact that the department where the head nurse attended none of the sessions registered the lowest vaccination rate. Promotion of vaccination by the head of the department has also been shown to determine the attitude of caregivers. ${ }^{5}$ Peer-to-peer influence was also demonstrated to be beneficial. In contrast, staff strongly opposed to vaccination had a negative impact on the rest of the group attending the session. Forty percent of those who refused the vaccine feared adverse events or expressed doubts on the safety of vaccination. The current debate in France concern- ing the safety of hepatitis B vaccine, frequently mentioned during the discussion part of the interventions, accounted for one-third of refusals and had a detrimental effect on those who hesitated. Nurses appeared less susceptible to such antivaccination attitudes. Improved targeting of information towards different staff categories could have a positive impact on vaccination rates. Easy access to vaccination appears sufficient to boost vaccination rates among medical staff. However, overall, it seems appropriate to provide information along with access to vaccination at the work site.

Our intervention proved effective but was time-consuming and required a mobile medical team. At present, vaccination is recommended for healthcare workers as a measure for protection against workplace risk and exposure, but emphasizing the role of vaccination in the prevention of nosocomial infections requires concerted action on the part of the occupational medicine department, hospital management, and personnel in charge of nosocomial infection control. Involving a senior member within each department to advocate immunization among the staff would be helpful. Lastly, the effects of repeated yearly immunization for 2-3 decades remain to be examined.

Providing information about and easy access to influenza vaccination for hospital personnel, therefore, should be promoted because of its medical and economic impact, as well as to ensure the sustained availability of able-bodied caregivers during influenza outbreaks in the community. Our results indicate that this type of intervention requires sustained commitment on the part of a mobile medical team, careful planning, and a coordinated effort on the part of the various departments concerned.

Brigitte Dunais, MD; Carine Saccomano, MD; Aline Mousnier, PharmD; Marie-Christine Roure, MD; Pierre Dellamonica, PhD; Pierre-Marie Roger, $\mathrm{PhD}$

From the Nice University Hospital, Nice, France (all authors).

Address reprint requests to Brigitte Dunais, Public Health Department, Hôpital de l'Archet 1, BP 3079, Nice cedex 3, 06202, France (dunais.b@chu -nice.fr).

Infect Control Hosp Epidemiol 2006; 27:529-531

(C) 2006 by The Society for Healthcare Epidemiology of America. All rights reserved. 0899-823X/2006/2705-0021\$15.00. 


\section{REFERENCES}

1. Viboud C, Pakdaman K, Boelle PY, et al. Association of influenza epidemics with global climate variability. Eur J Epidemiol 2004; 19:1055-1059.

2. Comité technique des vaccinations et section des maladies transmissibles. Avis des 19 et 25 Novembre 1999 du Conseil Supérieur d'Hygiène Publique de France concernant la vaccination contre la grippe. Bulletin officiel Solidarité-Santé 99/51. December 1999.

3. Carman WF, Elder AG, Wallace LA, et al. Effects of influenza vaccination of health care workers on mortality of elderly people in long-term care: a randomised control trial, Lancet 2000; 355:93-97.

4. Harbarth S, Siegriest CA, Schira JC, Wunderli W, Pittet D. Influenza immunization: improving compliance of health care workers. Infect Control Hosp Epidemiol 1998; 19:337-342.

5. Sartor C, Tissot-Dupont H, Zandotti C, Martin F, Roques P, Drancourt $M$. Use of a mobile cart influenza program for vaccination of hospital employees. Infect Control Hosp Epidemiol 2004; 25:918-922.

6. Yassi A, Murdzak C, Cheang M, Tran N, Aoki FY. Influenza immunization: knowledge, attitude and behaviour of health care workers. Can J Infect Control 1994; 9:101.

\section{On the Need for a Separate Standard for Performance Testing of Negative- Pressure Isolation Rooms}

TO THE EDITOR-All patient rooms in hospitals should provide an acceptable environment for patients to recover and a good working environment for the healthcare professionals who attend to them. The special purpose of a negativepressure isolation room is to protect healthcare workers, other patients, and visitors in a hospital from exposure to an airborne infectious agent in the event that an infectious patient is staying in the room. A principal design goal for a negativepressure isolation room, then, should be to achieve and maintain an adequate level of airborne infection protection in the environment surrounding an infectious patient-in other words, to contain the airborne infectious material in such a way that the threat of exposure to healthcare personnel in the isolation room and others outside the room is minimized. Isolation rooms are designed and constructed with this in mind, and their performance should be adequately tested to ensure that they function properly.

Performance testing can consist of a combination of measuring with permanent monitors and monitoring with transient testing methods- that is to say, monitoring with equipment that is used for a certain test or check and then removed. Performance testing can occur either before or as part of the commissioning of a new isolation room or as part of periodic maintenance. Performance testing of isolation suites can include monitoring of various parameters and events: pressure differentials between rooms, any pressure drops for high efficiency particulate air (HEPA) filters (particularly for extract registers, which can become clogged over time), the direction of airflow between rooms, within-room airflow patterns, the air exchange rate, the supply and exhaust air volumes and ventilation differential volume, envelope tightness (ie, the amount of leakage the isolation room has), containment, thermal comfort, and any leakage of installed HEPA filters.

In existing national guidelines that deal with control of airborne infection, discussions of elements of negative-pressure isolation room ventilation strategy and design tend to be sprinkled with advice, recommendations, and/or requirements regarding performance testing. For example, in the Centers for Disease Control and Prevention guidelines from $1994,{ }^{1}$ there are paragraphs describing smoke testing for observation of airflows within and between rooms and use of pressure-measuring devices in the section "Achieving Negative Pressure in a Room" (on p. 56), whereas recommendations for pressure-drop and leakage testing of HEPA filters are given in the "HEPA Filtration" section of "Supplement 3: Engineering Controls" (on p. 60). A fragmented picture emerges of what really needs to be done to determine whether the performance of a negative-pressure isolation room is good enough.

It is informative in this regard to look at performance testing of cleanrooms. Cleanrooms employ a form of protective isolation that is vital to the manufacture of pharmaceuticals. Ventilation engineers with a specialization in cleanrooms often also work on isolation room design. The published international standard for cleanrooms includes separate and specific parts dealing with design, ${ }^{2}$ performance testing methods and metrology, ${ }^{3}$ and specification of which tests and monitors are necessary to demonstrate continued satisfactory performance over time. ${ }^{4}$ Obviously, for relatively straightforward tests, such as those used to measure pressure differentials and the direction of airflow between rooms, there isn't a lot of uncertainty about the right way to do things (though there is a great disparity in national infection control standards regarding how much pressure difference is enough). For morecomplicated and specialized tests, however, there is a lack of guidance and consensus about how the tests should be done in isolation rooms and what the results should be. How to deal with more complicated testing would not be a serious issue if room pressure differentials and visualization of airflow patterns alone could adequately characterize isolation room containment and if ventilation system parameters never deviated from design values. Performance testing could then be limited to measurement of the pressure differentials at the time the room is commissioned and performance of some smoke tests to make sure flow is inward through door openings and mixing is good throughout the isolation room. Then the pressure differential could be monitored over time to verify that the differentials and airflow don't change, in conjunction with replacement of filters at recommended intervals. This is, in fact, the extent of performance testing of isolation rooms in many hospitals.

Unfortunately, system parameters always deviate from design values - if not at the start, then at some point during the service life of the system. And there is much evidence that there is more to containment than just maintenance of pressure differentials and visualization of smoke patterns. If 